\title{
Ecofácies e Processos Sedimentares em Plataforma Rasa: exemplo na Baia de Tijucas (Santa Catarina-Brasil)
}

Ricardo P. Meireles, Laboratório de Oceanografia Costeira, Universidade Federal de Santa Catarina, Brazil.

Antonio H. F. Klein, Laboratório de Oceanografia Costeira, Universidade Federal de Santa Catarina, Brazil.

Jair Weschenfelder, Instituto de Geociências, Universidade Federal do Rio Grande do Sul CECO/UFRGS, Brazil.

Jorge A. G. Souza, CB\&I - Environmental \& Infrastructure, Florianopólis, Brazil.

Reginaldo Lemos, Laboratório de Oceanografia Costeira, Universidade Federal de Santa Catarina, Brazil.

Andrew J. G. Cooper, School of Environmental Sciences. University of Ulster. Coleraine, North Ireland.

Elírio E. Toldo Jr., Instituto de Geociências, Universidade Federal do Rio Grande do Sul CECO/UFRGS, Brazil.

Copyright 2016, SBGf - Sociedade Brasileira de Geofísica

Este texto foi preparado para a apresentação no VII Simpósio Brasileiro de Geofísica, Ouro Preto, 25 a 27 de outubro de 2016. Seu conteúdo foi revisado pelo Comitê Técnico do VII SimBGf mas não necessariamente representa a opinião da SBGf ou de seus associados. É probida a reproducão total ou parcial deste material propósitos comerciais sem prévia autorização da SBGf.

\section{Abstract}

The Holocene shoreface and inner shelf of Tijucas bay, Santa Catarina - Brazil, is part of the extensive wavedominated, high sediment supply coast of Santa Catarina. The coast comprises accumulations of mixed sand-andmud representative of a series of muddy tidal flats, overbank deposits and cheniers. In particular, the inner shelf/shoreface is strongly heterogeneous in terms of composition. Such shorefaces are rare, under reported features. With this is mind, the aim of this study was to identified and classified echofacies in ultra resolution seismics profiles (CHIRP type), facies and depositional processes. The resultes reveal five echofacies. These are interpreted as fluid mud, seafloor, a Holocene barrier and a Quaternary sediments, respectively. The great diversity of mapped echofacies to suggest that the Tijucas Bay underwent different evolutionary sedimentary processes during the Holocene, to the establishment of its current geomorphological configuration

\section{Introdução}

Utilizando a geofísica marinha rasa, buscou-se desenvolver métodos para uma melhor classificação, em sub-superfície, do fundo marinho, e assim auxiliar no entendimento e na caracterização dos ambientes estudados. Diferentes respostas (e suas características) provenientes do sinal acústico juntamente com os distintos tipos de ecofácies (ecocaráter ou fácies acústicas), são relevantes quando se faz necessário a interpretação de processos de erosão e deposição em ambientes marinhos (Damuth, 1978). A ocorrência e distribuição de padrões sonográficos e de ecofácoies sísmicas de alta frequência têm relação estreita com a distribuição das características sedimentares nos diferentes ambientes de sedimentação (Ayres Neto, 2000; Weschenfelder et al., 2005; Souza, 2006; Pereira et al., 2009). Desta forma, esses padrões podem ser utilizados como ferramenta para auxiliar na definição e distinção dos processos sedimentares que atuaram na formação de um determinado pacote sedimentar. Isso se deve ao fato de que a interação dos sedimentos com o meio de transporte resulta na formação de formas de leito (bedforms), as quais podem ser preservadas como estruturas em rochas sedimentares e, portanto, fornecer um registro dos processos deposicionais que ocorreram no momento da sedimentação (Nichols, 2009).

Os depósitos do Holoceno da plataforma interna da baia de Tijucas em Santa Catarina - Brasil, foram acumulados em sistema de energia mista, dominado por ondas e com altas taxas de sedimentação, alimentados principalmente pelo Rio Tijucas. Na planície são descritos os únicos depósitos de chêniers do Estado de Santa Catarina (SC) (Hein et al., 2015). Na plataforma interna foram acumulados sedimentos com misturas granulométricas, entre areias e lamas, o que caracteriza esse sistema plataforma/planície costeira como fortemente heterogêneo em termos de sua composição (Cooper et al., submetido).

Utilizando registros sísmicos de alta resolução (tipo CHIRP), este trabalho tem como objetivo descrever as ecofácies e discutir os principais processos deposicionais que atuaram durante 0 desenvolvimento desses depósitos, majoritariamente Holocênicos, para buscar maior entendimento da evolução no contexto paleoambiental e auxiliar estudos sobre taxas de sedimentação e variações relativas do nível do mar.

\section{Área de Estudo}

A área de estudo abrange a baia de Tijucas, situada no Município homônimo no litoral centro-norte do Estado de Santa Catarina $\left(27^{\circ} 15^{\prime} \mathrm{S} ; 48^{\circ} 37^{\prime} \mathrm{W}\right)$, distante $50 \mathrm{~km}$ da capital Florianópolis. Tijucas possui uma área de aproximadamente $280 \mathrm{~km}^{2}$ e sua planície costeira se estende por $12 \mathrm{~km}$ × $6 \mathrm{~km}$ (longitudinal e perpendicular respectivamente), abrigada por promontórios rochosos, com clima de ondas que variam entre 0,3 à $0,5 \mathrm{~m}$ em regime de micromaré (Schettini et al., 2010) (Fig. 1).

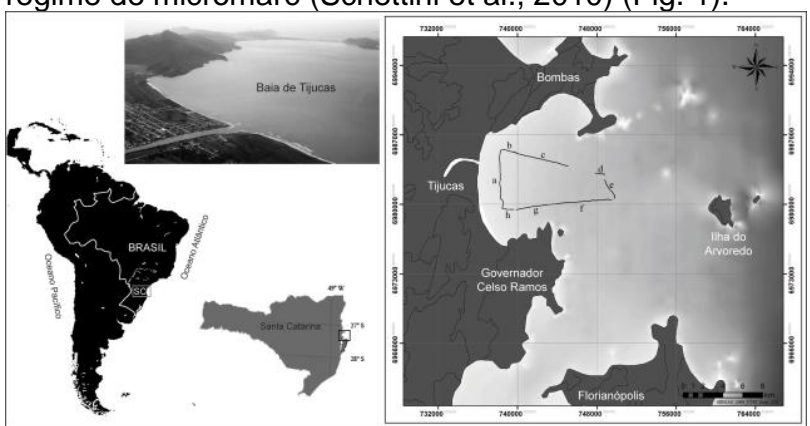

Figura 1 - Mapa de localização da área de estudo com destaque para as linhas sísmicas $a, b, c, d, e, f, g, h$. 


\section{Métodos}

O trabalho foi desenvolvido em 3 (três) fases principais: (1) levantamento geofísico, (2) processamento e (3) interpretação e determinação das ecofácies, com respectiva vinculação aos processos sedimentares. O levantamento geofísico foi realizado com o auxílio de um perfilador sísmico de alta resolução tipo $\mathrm{CHIRP \AA}$ da marca EdgeTech 3200 modelo SB 216-S; a aquisição foi feita com uma taxa de amostragem de 5 milissegundos e frequência modulada de 1 a $10 \mathrm{kHz}$.

Para garantir uma precisão submétrica no posicionamento da embarcação e consequentemente dos equipamentos utilizados no levantamento, utilizou-se um DGPS (Differential Global Positioning System) marca Trimble®, modelo SPS282, com sinal diferencial fornecido pelo sistema OmniStar® VBS.

Os dados sísmicos foram gravados originalmente no formato próprio da ODEC e posteriormente transformados para o formato SEG-Y, para posterior processamento e interpretações.

$\mathrm{Na}$ etapa (2) foram processados os dados digitais, nos softwares livres SeisPrho® (versão 2.0), e SeiSee® (versão 2.22.2) e a velocidade de propagação do som utilizada foi de $1.550 \mathrm{~m} / \mathrm{s}$. A etapa (3) consistiu na interpretação dos perfis sísmicos, que permitiram a identificação de diferentes ecofácies sísmicas, indicativos de distintos processos sedimentares.

A identificação dos tipos de ecofácies foi feita mediante a análise de parâmetros como: configuração, amplitude e frequência, continuidade lateral e arranjo das reflexões sísmicas, baseados em estudos de caso. O gás ocorre disperso em quase todas as fácies e neste trabalho foi considerado um tipo de ecofácie. As múltiplas também foram consideradas por estar relacionadas a depósitos arenosos.

\section{Resultados}

A análise sísmica nos dados permitiu a individualização de 6 (seis) ecofácies ou ecocaracteres, definidos pelas suas características acústicas e padrões geométricos, que podem estar associados aos processos sedimentares e à morfologia do fundo da baia de Tijucas, Tabela 1 e Figura 2. Os diferentes tipos de ecofácies foram reconhecidos e classificados seguindo, de maneira geral, as proposições de Baptista Neto et al. (1996), Quaresma et al. (2000) e Catanzaro et al. (2004), com adaptações.

\section{Ecofácies Tipo I}

Refere-se à interface água-sedimento de fundo. Apresenta sinal de retorno intenso, amplitude média (10) relativa e alta frequência (770); os refletores ocorrem sobre superfície regular de forma continua e horizontal. A ecofácie Tipo I é o topo da unidade estratigráfica U1, composta por sedimentos essencialmente lamosos e inconsolidados de perto da costa. Em perfis sísmicos perpendiculares a linha de costa, esses refletores ocorrem em profundidades médias que variam de $-3 \mathrm{~m}$ à -11 m (Tabela 1 e Fig. 2).

\section{Ecofácies Tipo II}

Apresenta amplitude média relativa, alta frequência e sinal de retorno intenso. As reflexões são continuas e concordantes com a ecofácie Tipo I. O sinal acústico é atenuado do topo para a base, variando de alta intensidade no refletor de topo para baixa no refletor de base desta ecofácie. Esses refletores ocorrem em profundidades médias que variam de $-5 \mathrm{~m}$ à $-8 \mathrm{~m}$ e em áreas mais profundas da baia em profundidades entre $11 \mathrm{~m}$ à -13 m (Tabela 1 e Fig. 2).

\section{Ecofácies Tipo III}

Apresenta sinal de retorno variando de média à alta intensidade, amplitudes relativamente altas e baixa frequência. Os refletores ocorrem de forma segmentada ou descontínua, geralmente preenchendo estruturas de corte/preenchimento, com suaves mergulhos sobre o pacote inferior relacionado à ecofácie Tipo IV. Esses refletores ocorrem em profundidades médias que variam de $-15 \mathrm{~m}$ à $-19 \mathrm{~m}$ e em áreas mais profundas da baia em profundidades entre $-25 \mathrm{~m}$ à $-36 \mathrm{~m}$ (Tabela 1 e Fig. 2).

\section{Ecofácies Tipo IV}

Expressa um sinal de retorno variando de baixa a media intensidade, amplitude média relativa e baixa frequência. Tais refletores também ocorrem de forma segmentada ou descontínua com suaves mergulhos. Nestas ecofácies ocorrem refletores que delineiam a forma de canais. Esses refletores ocorrem em profundidades médias que variam de $-29 \mathrm{~m}$ à $-36 \mathrm{~m}$ (Tabela 1 e Fig. 2).

\section{Ecofácies Tipo $V$}

A ecofácie Tipo $V$ expressa um sinal de alta intensidade, alta frequência e amplitude média relativa. Está associado a acúmulos de gás nos sedimentos, identificados como feições do tipo (a) cobertura acústica, (b) plumas intra-sedimentares e (c) pináculos de turbidez (Tabela 1 e Fig. 2). São registrados em toda à área de estudo (Fig. 2) (ver Klein et al., 2016 submetido e Weschenfelder et al., 2015 para mais informações)

\section{Ecofácies Tipo VI}

As 'múltiplas', não tem característica própria que as definam como uma ecofácie sísmica, porém tais refletores repetem as mesmas morfologias dos refletores primários, variando a amplitude e frequência, configurando assim um ruído nos dados sísmicos e por este motivo foram consideradas neste trabalho como Tipo IV. Foram encontradas múltiplas de primeira ordem, geradas a partir da interface água/sedimento e múltiplas de segunda ordem (internas), geradas nas interfaces entre camadas (ver Luz, 2012 para mais informações) (Tabela 1 e Fig. 2).

\section{Discussões \& Conclusões}

De maneira geral, os processos sedimentares que atuaram e/ou atuam na plataforma rasa na baia de Tijucas estão relacionados à alternância de paleoambientes e do regime de energia. Foram reconhecidas e individualizadas 06 fácies deposicionais, detalhadas a seguir:

Fácies I - Compreende a camada mais superficial (centímetros até o $1^{\circ}$ metro de deposição) e apresenta distribuição planar no registro sísmico, correspondendo à ecofácies Tipo I. Composta essencialmente por lama compacta sotoposta a lama fluida, depositada essencialmente em condições marinhas de baixa energia, pouco perturbada. A gênese deste depósito de sedimentos finos recentes está intimamente relacionada 
ao aporte fluvial para dentro da baia, neste caso o rio Tijucas.

Fácies II - Depósitos sedimentares métricos associados ao ecofácies Tipo II, com arquitetura deposicional formando laminações paralelas, composta principalmente por lama compacta, depositada essencialmente em paleoambiente marinho restrito com influência da descarga fluvial que favoreceu o desenvolvimento e acúmulo de gás.

Fáceis III - Corresponde a depósitos sedimentares de espessuras métricas associados à ecofácies Tipo III. Este conjunto de fácies arquitetônicas é classificado como leito arenoso (barreira) com grande dominância de estratificação horizontal e planar. A presença de sequências de fácies arenosas, com mergulho dos refletores, evidencia o balanço entre o fluxo de energia e a profundidade, atribuindo a esta deposição um paleoambiente marinho raso-transicional acrescido pelos processos de by-pass (produzido pela deriva litorânea); e overstep (Cooper et al., submetido).

Fácies IV - Corresponde à superfície erosiva na base da deposição Holocênica, caracterizado por fácies arenosas indiferenciadas depositadas durante a regressão do Pleistoceno e associadas à ecofácie Tipo IV.

Fácies $\boldsymbol{V}$ - Corresponde à resposta acústica produzida pelo gás disseminado nos poros do pacote sedimentar. $\mathrm{O}$ gás intersticial produz uma resposta acústica anômala, de arquitetura variada nos ecogramas, cujas características são aqui associadas à ecofácie Tipo V.

As ecofácies Tipos I e II estão corelacionados a uma superfície continua e homogênea, sendo indicativos de um ambiente estável e de baixa energia, que propiciou uma taxa de sedimentação constante ou a não deposição e sim retrabalhamento dos sedimentos, ocasionado pelas ondas.

As ecofácies Tipos III e IV estão correlacionados às superfícies erosivas, ocorrem de forma oblíqua, segmentada ou descontinua, evidenciando um ambiente sujeito a variações de energia de deposição e/ou ciclos erosivos.

A grande diversidade de ecofácies mapeadas permite afirmar que a Baia de Tijucas passou por distintos processos sedimentares evolutivos durante o Holoceno, até 0 estabelecimento de sua configuração geomorfológica atual.

\section{Agradecimentos}

Ao Conselho Nacional de Desenvolvimento Científico e Tecnológico (CNPq - processo: 400542/2013-7); PósDoc BJT/CNPq (processo: 401759/2013-0); Bolsa de Produtividade em Pesquisa do CNPq (Nível 2 - CA OC Oceanografia; processo 313777/2013-6); ao Programa Petrobras de Desenvolvimento de Recursos Humanos PFRH 240. A empresa CB\&I e ao Oc. Rodrigo Cavalheiro (gerência de Florianópolis) pela aquisição e suporte técnico durante o levantamento. A Empresa Cultura SubAquática pelo suporte logístico da embarcação. Ao Dr. Andrew Green pelas discussões.

\section{Referências}

Ayres Neto, A. N., 2000. Uso da sísmica de reflexão de alta resolução e da sonografia na exploração mineral submarina. Brazilian Journal of Geophysics, 18(3): 241 256.

Baptista Neto, J. A., Silva, M. A. M. \& Figueiredo Jr., A. G., 1996. Sísmica de alta frequência e o padrão de distribuição de sedimentos na Enseada de Jurujuba (Baía de Guanabara) - RJ/Brasil. Revista Brasileira de Geofísica, 14(1): 51-58.

Catanzaro, L. F., Baptista Neto, J. A., Guimarães, M. S. D. \& Silva, C. G., 2004. Distinctive sedimentary processes in Guanabara bay - SE/Brazil, based on the analysis of echo-character $(7.0 \mathrm{kHz})$. Revista Brasileira de Geofísica, 22(1): 69-83.

Cooper et al. (07 co-authors). Sandy barrier overstepping and preservation under rapid sea level rise: an example from southern Brazil. Submetido.

Damuth, J. E., 1978. Echo character of the Norwegian Greenland sea - relationship to quaternary sedimentation. Marine Geology, 28: 1-36.

Hein et al. (05 co-authors), 2015. Wave energy, sediment supply, and sea-level fall: late Holocene basin infilling in Southern Brazil. Coastal Sediments 2015: The Proceedings of the Coastal Sediments. 1-16.

Nichols, G., 2009. Sedimentology and stratigraphy. WileyBlackwel, 2nd ed. 419p.

Pereira, M. C., Weschenfelder, J. \& Corrêa, I. C. S., 2009. Reconhecimento e mapeamento de fácies sísmicas nos pontais arenosos da lagoa dos Patos, RS, Brasil. Pesquisas em Geociências, 36(1): 23-35.

Quaresma V. S, Dias, G. T. M \& Baptista Neto, J. A., 2000. Caracterização da ocorrência de padrões de sonar de varredura lateral e sísmica de alta frequência (3,5 e $7,0 \mathrm{kHz}$ ) na porção sul da Baía de Guanabara - RJ. Revista Brasileira de Geofísica, 18(2): 201-214.

Schettini, C. A. F., Almeida, D. C., Siegle, E. \& Alencar, A. C. B., 2010. A snapshot of suspended sediment and fluid mud occurrence in a mixed-energy embayment, Tijucas Bay, Brazil. Geo-Marire Letter, 30: 47-62.

Souza, L. A. P., 2006. Revisão crítica da aplicabilidade dos métodos geofísicos na investigação de áreas submersas rasas. Tese de Doutorado, Instituto Oceanográfico da USP, São Paulo - SP, 311p.

Weschenfelder, J., Corrêa, I. C. S. \& Aliotta, S., 2005. Elementos arquiteturais do substrato da Lagoa dos patos revelados por sísmica de alta resolução. Pesquisas em Geociências, 32(2): 57-67. 


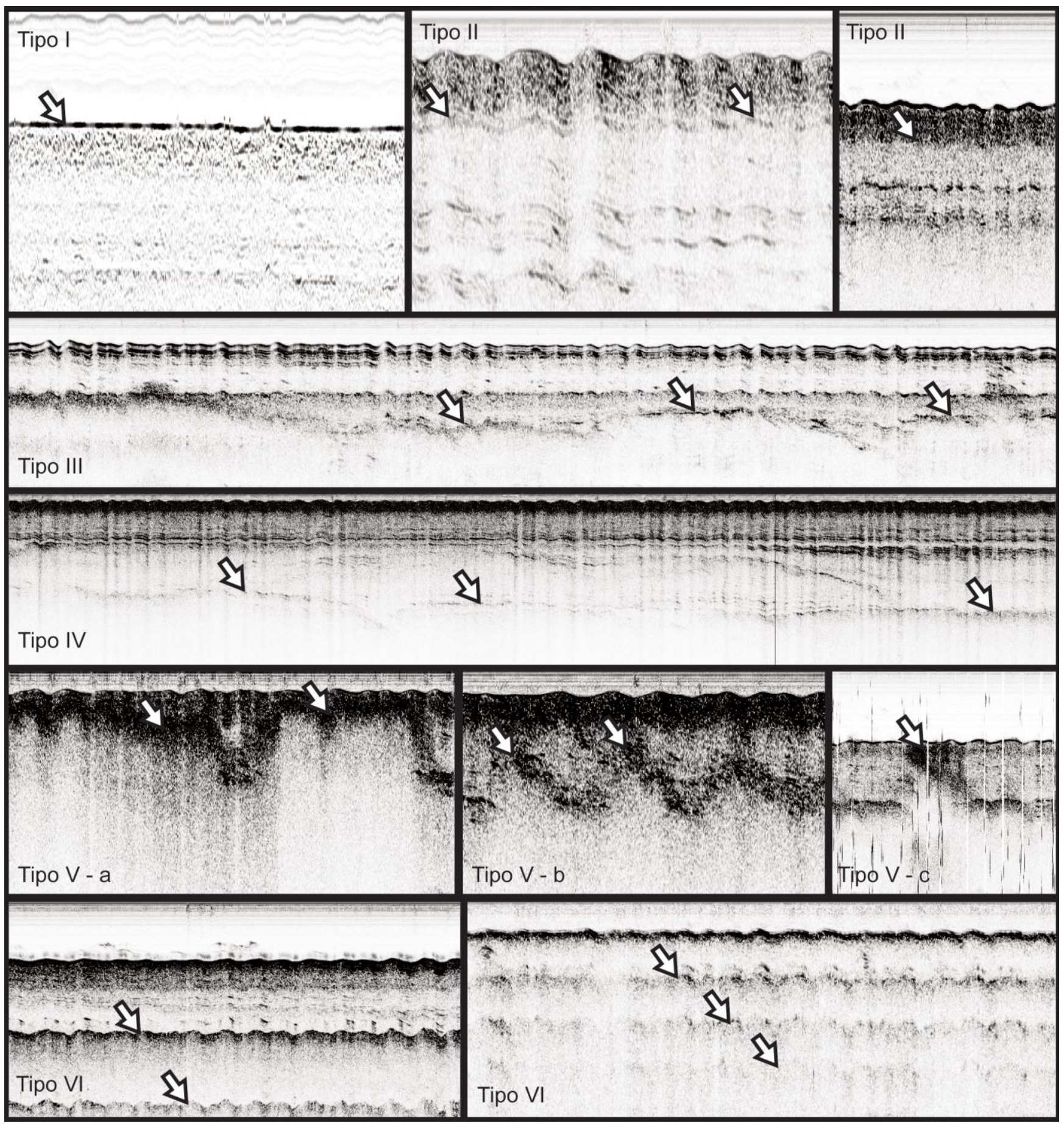

Figura 2-Tipos (I, II, III, IV, V e VI) de ecofácies sísmicas observados na baia de Tijucas, Santa Catarina, Brasil. 
Tabela 1 - Tipos e características das ecofácies sísmicas encontrados na baía de Tijucas.

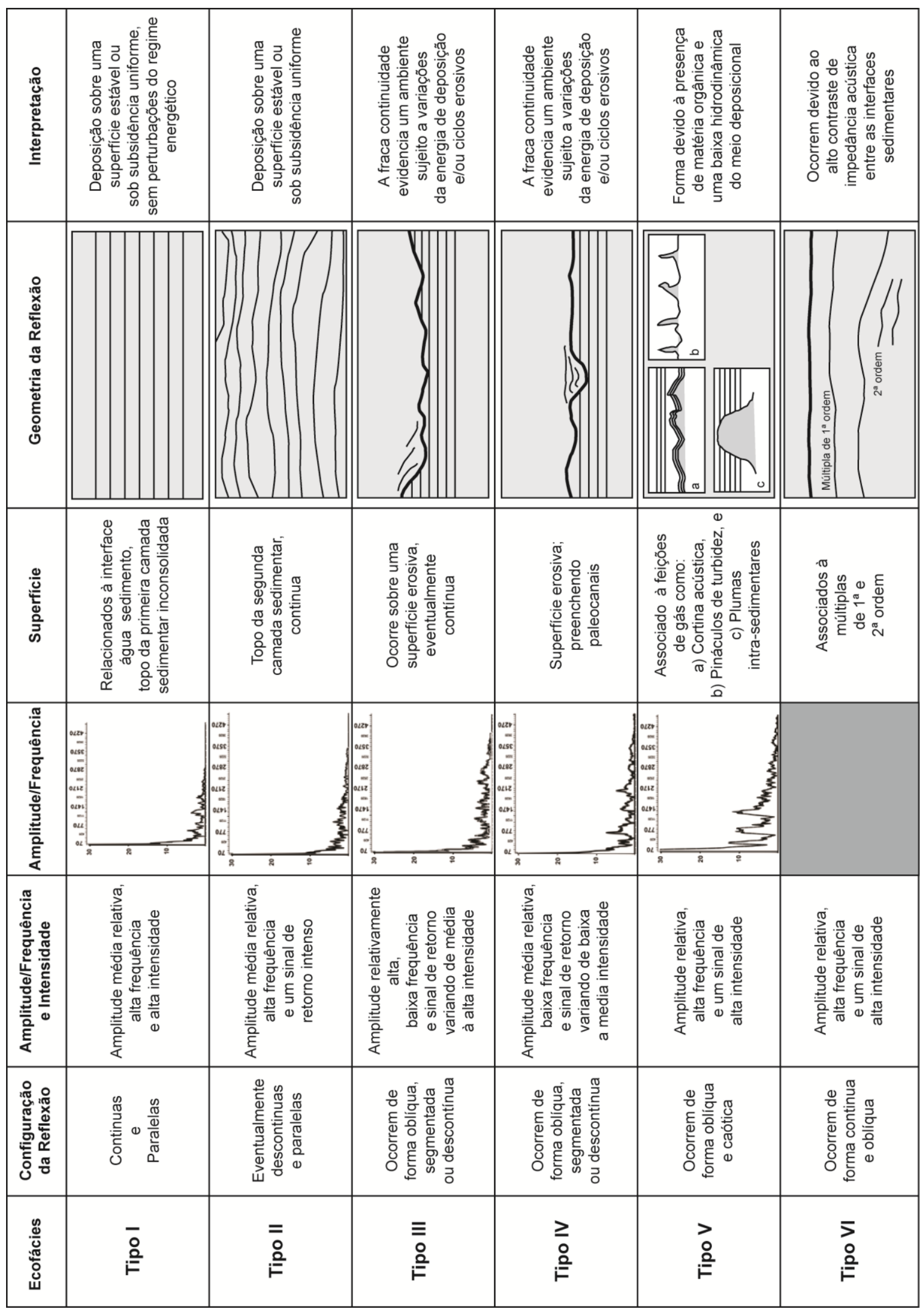

